\title{
INTEGRAL REPRESENTATIONS OF CYCLIC GROUPS OF ORDER $p^{2}$
}

\author{
IRVING REINER ${ }^{1}$
}

\begin{abstract}
Let $p$ be an odd prime, either regular or properly irregular, and let $G$ be a cyclic group of order $p^{2}$. The author determines a full set of inequivalent representations of $G$ by matrices with rational integral entries. This information is used to calculate the ideal class number of the integral group ring $Z G$.
\end{abstract}

Let $G$ be a cyclic group of order $p^{2}$ with generator $x$, where $p$ is an odd prime. We shall determine the number $n(Z G)$ of nonisomorphic indecomposable $Z G$-lattices, for the case where $p$ is a regular odd prime (that is, $p$ does not divide the class number of the cyclotomic field $\left.Q\left({ }^{p} \vee 1\right)\right)$. We shall also calculate $h(Z G)$, the number of isomorphism classes of ideals $M$ in $Z G$ for which $Q M=Q G$. Such problems were considered previously by various authors (see Heller-Reiner [2], for example), but no explicit formulas for $n(Z G)$ and $h(Z G)$ were obtained, because of difficulties about units. The methods of Galovich [1] enable one to overcome these difficulties for the case where $p$ is a regular odd prime. We restrict ourselves to this case from now on, unless otherwise stated.

We may write the integral group ring $Z G$ as $Z[x] /\left(x^{p^{2}}-1\right)$. Letting $\phi_{p^{m}}(x)$ denote the cyclotomic polynomial of order $p^{m}$, we now set

$$
R=Z[x] /\left(\phi_{p}(x)\right), \quad S=Z[x] /\left(\phi_{p^{2}}(x)\right), \quad T=Z[x] /\left(x^{p}-1\right) .
$$

Then $R$ is the ring of all algebraic integers in the cyclotomic field $Q\left({ }^{P} \vee 1\right)$; let $h_{R}$ denote its class number. Likewise, $S$ is the ring of integers in $Q\left({ }^{P^{2}} \vee 1\right)$; let its class number be $h_{S}$. Finally, $T$ is the integral group ring of a group of order $p$.

We shall set $\lambda=1-x$, viewed either as an element of $Z G$, or else as an element of some quotient ring, such as $R, S, R / p R$, and so on. Let

$$
\begin{gathered}
\bar{Z}=Z / p Z, \quad \bar{R}=R / p R=R / \lambda^{p-1} R \cong \bar{Z}[\lambda] /\left(\lambda^{p-1}\right), \\
\bar{T}=T / p T \cong \bar{Z}[\lambda] /\left(\lambda^{p}\right) .
\end{gathered}
$$

The subscript $p$ will indicate $p$-adic completion. In order to conform to the notation in [2], we now set

$$
A=Z_{p} a \cong Z_{p}, \quad B=R_{p} b \cong R_{p}, \quad C=S_{p} c \cong S_{p}, \quad E=T_{p} e \cong T_{p},
$$

where $A, B, C$ and $E$ are viewed as $Z_{p} G$-lattices. For each $Z G$-lattice $N$, we have

Received by the editors August 25, 1975.

AMS (MOS) subject classifications (1970). Primary 20C05, 20C10; Secondary 12A45.

${ }^{1}$ This work was supported by a research grant from the National Science Foundation. 


$$
\operatorname{Ext}_{Z G}^{1}(S, N) \cong \operatorname{Ext}_{Z_{p} G}^{1}\left(S_{p}, N_{p}\right) \cong \bar{N}=N / p N .
$$

As shown in [2], a full list of nonisomorphic indecomposable $Z_{p} G$-lattices is as follows:

(1) $A, B, C, E$.

(2) Extensions of $C$ by $A \oplus E$, corresponding to extension classes $\bar{a}+\lambda^{r} \bar{e}$ in $\bar{A} \oplus \bar{E}, 1 \leqslant r \leqslant p-2$.

(3) Extensions of $C$ by $E$, corresponding to extension classes $\lambda^{r} \cdot \bar{e}, 0 \leqslant r$ $\leqslant p-1$.

(4) Extensions of $C$ by $A \oplus B$; classes $\bar{a}+\lambda^{r} \bar{b}, 0 \leqslant r \leqslant p-2$.

(5) The extension of $C$ by $A$, with extension class $\bar{a}$.

(6) Extensions of $C$ by $B$, corresponding to extension classes $\lambda^{r} \bar{b}, 0 \leqslant r$ $\leqslant p-2$.

For each indecomposable $Z G$-lattice $X$, we know from [2] that its completion $X_{p}$ is also indecomposable, and hence occurs in the above list. We shall say that $X$ is of "type $k$ " if $X_{p}$ is listed in the $k$ th grouping. Corresponding to the $Z_{p} G$-lattice $A$, the only possible $X$ is the $G$-trivial module $Z$. Next, the $h_{R}$ representatives of the ideal classes of $R$ give (up to isomorphism) all indecomposable $Z G$-lattices $X$ for which $X_{p} \cong B$. Likewise, there are $h_{S}$ ideals of $S$ which correspond to $C$. Finally, there are precisely $h_{R}$ possible $X$ 's corresponding to $T$, namely, the projective ideals of the integral group ring $T$. (This last assertion follows readily from the discussion below.)

Consider next an $X$ of type (6), so $X_{p}$ is an extension of $C$ by $B$. Then $X$ must be isomorphic to an extension of $\mathfrak{c}$ by $\mathfrak{b}$, where $\mathfrak{c}$ is an ideal of $S$, and $\mathfrak{b}$ is an ideal of $R$. Now

$$
\operatorname{Ext}_{Z G}^{1}(\mathfrak{c}, \mathfrak{b}) \cong \operatorname{Ext}_{z G}^{1}(S, R) \cong \bar{R}
$$

Furthermore,

$$
\text { Aut } \mathrm{c} \cong \text { Aut } S \cong u(S), \quad \text { Aut } \mathfrak{b} \cong \text { Aut } R \cong u(R),
$$

where Aut denotes the group of $Z G$-automorphisms, and $u()$ denotes the group of units. However, the isomorphism class of $X$ is completely determined by the orbit of its extension class under the action of Aut $c$ and Aut $\mathfrak{b}$. It follows at once that in enumerating such orbits, we need only consider the case where $X$ is an extension of $S$ by $R$. The total number of $X$ 's will then be $h_{R} h_{S}$ times the number of extensions of $S$ by $R$.

So now let $X$ be an extension of $S$ by $R$, and let $\xi \in \bar{R}$ be its class in $\operatorname{Ext}^{1}(S, R)$. We may write $\xi=\lambda^{r} v$, where $0 \leqslant r \leqslant p-2$ and where $v$ is a unit in the ring $R^{\prime}=\bar{Z}[\lambda] /\left(\lambda^{p-1-r}\right)$. We must calculate the number of orbits of $u\left(R^{\prime}\right)$ under the action of $u(R)$ and $u(S)$. For each $w \in u(S)$, its norm $N(w)$ from $S$ to $R$ is a unit of $R$. It is easily verified that $w$ acts on $u\left(R^{\prime}\right)$ in the same way that $N(w)$ acts. Hence it suffices for us to count the orbits of $u\left(R^{\prime}\right)$ under the action of $u(R)$.

Now $u\left(R^{\prime}\right)$ is the direct product of a cyclic group $D$ of order $p-1$, and an elementary abelian $p$-group generated by the units

$$
1+\lambda, 1+\lambda^{2}, 1+\lambda^{3}, \ldots, 1+\lambda^{p-2-r} .
$$

But for $p$ regular, Galovich [1] showed that the image of $u(R)$ in $u\left(R^{\prime}\right)$ is the product of $D$ and a subgroup generated by units of the form 


$$
\begin{aligned}
1+\lambda, 1+\lambda^{2}+\alpha_{2} \lambda^{3}, 1+ & \lambda^{4}+\alpha_{4} \lambda^{5}, \\
& 1+\lambda^{6}+\alpha_{6} \lambda^{7}, \ldots, 1+\lambda^{k}+\alpha_{k} \lambda^{k+1},
\end{aligned}
$$

where each $\alpha_{i} \in R^{\prime}$, and where $k$ is the largest even integer $\leqslant p-2-r$. The "missing" exponents are $3,5,7, \ldots, p-2-r$, the last term occurring only for even $r$. The number of such missing exponents is $[(p-3-r) / 2]$, where this symbol ${ }^{2}$ is interpreted as 0 whenever $p-3-r<0$. Consequently, the cokernel of the map $u(R) \rightarrow u\left(R^{\prime}\right)$ is an elementary $p$-group on $[(p-3-r) / 2]$ generators. Hence the total number of indecomposable $Z G$ lattices of type (6) is given by

$$
h_{R} h_{S} \alpha, \quad \text { where } \alpha=\sum_{r=0}^{p-2} p^{[(p-3-r) / 2]} .
$$

A similar argument shows that the number of lattices of type (4) is also given by formula (7). In this situation, we must count the orbits of $\bar{Z} \oplus \bar{R}$ under the action of $u(R)$ and $u(S)$. This action is given by

$$
w(\bar{z}+\bar{r}) u=N(w) \bar{z}+N(w) \bar{r} u
$$

for $w \in u(S), \bar{z}+\bar{r} \in \bar{Z} \oplus \bar{R}, u \in u(R)$. We may choose $w$ so that $N(w) \bar{z}$ $=\overline{1}$, and then proceed with the remaining term $N(w) \bar{r} u$ as in the preceding paragraph. This gives formula (7) just as before.

It is easily seen that there are $h_{S}$ indecomposable $Z G$-lattices of type (5), and it remains for us to consider lattices of types (2) and (3). Suppose that $X$ is of type (3), and is an extension of $S$ by $T$. We have seen that

$$
\operatorname{Ext}^{1}(S, T) \cong \bar{T} \cong \bar{Z}[\lambda] /\left(\lambda^{p}\right),
$$

and the extension class of $X$ is of the form $\lambda^{n} v$ for some $r, 0 \leqslant r \leqslant p-1$, and some $v \in u\left(R^{\prime}\right)$ with $R^{\prime}=\bar{Z}[\lambda] /\left(\lambda^{p-r}\right)$. We wish to count the number of orbits of $u\left(R^{\prime}\right)$ under the action of $u(S)$ and $u(T)$.

If $r \geqslant 1$ then $R^{\prime}$ is a quotient of $R$, and both $u(S)$ and $u(T)$ act on $R^{\prime}$ via $u(R)$. Hence by the preceding discussion, the number of orbits equals $p^{[(p-2-r) / 2]}$. Consider now the case $r=0$, in which $R^{\prime}=\bar{Z}[\lambda] /\left(\lambda^{p}\right)=\bar{T}$. The group $u(\bar{T})$ is the direct product of a cyclic group $D$ of order $p-1$, and an elementary $p$-group with generators

$$
1+\lambda, 1+\lambda^{2}, 1+\lambda^{3}, \ldots, 1+\lambda^{p-1} .
$$

On the other hand, we note that

$$
\bar{T}=Z[x] /\left(\phi_{p^{2}}(x), x^{p}-1\right) \cong S /\left(x^{p}-1\right)=S /\left(\lambda^{p}\right) .
$$

By [1], the image of $u(S)$ in $u(\bar{T})$ is the product of $D$ and the subgroup generated by units of the form

$$
1+\lambda, 1+\lambda^{2}+\alpha_{2} \lambda^{3}, 1+\lambda^{4}+\alpha_{4} \lambda^{5}, \ldots, 1+\lambda^{p-1},
$$

where each $\alpha_{i} \in \bar{T}$. The "missing" exponents are 3, 5, ., p-2; there are $(p-3) / 2$ such exponents. Furthermore, none of them can be recaptured from the action of $u(T)$ on $u(\bar{T})$, since each unit of $T$ maps onto a unit of $R$ under the surjection $T \rightarrow R$. Thus the number of orbits for the case $r=0$ is

${ }^{2}$ For $y \geqslant 0$, the symbol $[y]$ denotes the greatest integer $\leqslant y$. 
precisely $p^{(p-3) / 2}$. It follows at once that the total number of indecomposable $Z G$-lattices of type (3) is given by

$$
h_{R} h_{S} \beta, \quad \text { where } \beta=\sum_{r=0}^{p-1} p^{[(p-2-r) / 2]} .
$$

A similar argument shows that the number of lattices of type (2) is given by $(\alpha-1) h_{R} h_{S}$, since in (2) the parameter $r$ ranges from 1 to $p-2$. We may remark that

$$
\beta=\alpha+p^{(p-3) / 2},
$$

where $\alpha$ is defined in (7).

Combining all of these results, we have:

THEOREM. For $p$ a regular odd prime, the number $n(Z G)$ of isomorphism classes of indecomposable ZG-lattices is given by

$$
n(Z G)=1+2 h_{R}+2 h_{S}+(3 \alpha-1) h_{R} h_{S}+\beta h_{R} h_{S},
$$

where $\alpha, \beta$ are defined in (7) and (8).

We are now in a position to compute $h(Z G)$, the number of isomorphism classes of ideals $M$ in $Z G$ for which $Q M=Q G$. In the first column below, we list all $M$ 's (apart from using ideals in $R$ and $S$, rather than the rings $R$ and $S$ themselves). In the second column, we list the number of $M$ 's of each kind, omitting the factor $h_{R} h_{S}$ arising from the ideal classes.

\begin{tabular}{cc} 
Classes of ideals in $Z G$ & Number of classes \\
\hline$Z \oplus R \oplus S$ & 1 \\
$Z \oplus$ type 6 & $\alpha$ \\
$R \oplus$ type 5 & 1 \\
$S \oplus E$ & 1 \\
type 3 & $\beta$ \\
type 4 & $\alpha$
\end{tabular}

(Note that type 2 does not give ideals in $Z G$.) We thus obtain:

COROLlary. The number of ideal classes of $Z G$ is

$$
h(Z G)=(3+2 \alpha+\beta) h_{R} h_{S}
$$

with $\alpha, \beta$ as in (7) and (8).

When the prime $p$ is irregular, the expressions in the Theorem and its Corollary give lower bounds for $n(Z G)$ and $h(Z G)$, respectively. This follows from our earlier discussion and Galovich's results about units for the case of irregular primes.

The prime $p$ is called properly irregular if $p$ divides $h_{R}$, but does not divide the class number of the maximal real subfield of $Q\left({ }^{p} \vee 1\right)$. As pointed out by Galovich, his methods can be applied to the case of properly irregular primes to obtain analogues of the preceding Theorem and its Corollary. For $k$ $\leqslant(p-3) / 2$, let $\delta(k)$ be the number of Bernoulli numbers among $B_{1}$, $B_{2}, \ldots, B_{k}$ whose numerators are multiples of $p$. (If $p$ is a regular prime, each 
$\delta(k)=0$.) Assuming now that $p$ is properly irregular, let us consider the orbits of $u\left(R^{\prime}\right)$ under the action of $u(R)$, where $R^{\prime}=\bar{Z}[\lambda] /\left(\lambda^{p-1-r}\right)$, with $0 \leqslant r \leqslant p-2$. In determining the image of $u(R)$ in $u\left(R^{\prime}\right)$, we must omit from the list (6a) all those terms $1+\lambda^{2 i}+\alpha_{i} \lambda^{2 i+1}$ for which $p$ divides the numerator of $B_{i}$. It follows at once that the number of indecomposable $Z G$-lattices of type (6) is given by

$$
h_{R} h_{S} \alpha^{\prime}, \quad \text { where } \alpha^{\prime}=\sum_{r=0}^{p-2} p^{[(p-3-r) / 2]+\delta[(p-2-r) / 2]} .
$$

This also gives the number of indecomposables of type (4).

On the other hand, the image of $u(S)$ in $u(\bar{T})$ still contains $1+\lambda^{p-1}$, even when $p$ is properly irregular. It follows easily that the number of indecomposable lattices of type (3) is given by

$$
h_{R} h_{S} \beta^{\prime}, \quad \text { where } \beta^{\prime}=\alpha^{\prime}+p^{(p-3) / 2+\delta((p-3) / 2)} \text {. }
$$

Finally, the number of indecomposables of type (2) is given by $h_{R} h_{S}\left(\alpha^{\prime}-1\right)$. Thus, when $p$ is properly irregular, the analogues of the Theorem and its Corollary are valid, provided that we use $\alpha^{\prime}$ in place of $\alpha$, and $\beta^{\prime}$ in place of $\beta$.

As a concluding remark, it should be mentioned that in all cases known so far, every odd prime is either regular or properly irregular.

\section{REFERENCES}

1. S. Galovich, The class group of a cyclic p-group, J. Algebra 30 (1974), 368-387.

2. A. Heller and I. Reiner, Representations of cyclic groups in rings of integers. I, Ann. of Math.

(2) 76 (1962), 73-92. MR 25 \#3993.

Department of Mathematics, University of Illinois, Urbana, Illinois 61801 